Numer i cal anal ysi s of act i ve magnet i c r egener at or $\mathrm{S}$ f or hydr ogen magnet i $\mathrm{c}$ r ef ri ger at i on bet ween 20 and $77 \mathrm{~K}$

\begin{tabular}{|l|l|}
\hline 著者 & $\begin{array}{l}\text { Nat suntot o Koi chi, Kondo Takuya, I keda } \\
\text { Nasakazu, Numazawa Takenor i }\end{array}$ \\
\hline $\begin{array}{l}\text { j our nal or } \\
\text { publ i cat i on ti t l e }\end{array}$ & Cryogeni cs \\
\hline vol une & 51 \\
\hline number & 6 \\
\hline page range & $353-357$ \\
\hline year & $2011-06-01$ \\
\hline URL & ht t p: //hdl . handl e. net /2297/26242 \\
\hline
\end{tabular}




\title{
Numerical analysis of active magnetic regenerators for hydrogen magnetic refrigeration between 20 and $77 \mathrm{~K}$
}

\author{
Koichi Matsumoto ${ }^{\mathrm{a}, *}$, Takuya Kondo ${ }^{\mathrm{a}, 1}$, Masakazu Ikeda ${ }^{\mathrm{a}, 2}$, \\ Takenori Numazawa ${ }^{\mathrm{b}}$ \\ ${ }^{a}$ Department of Physics, Kanazawa University, Kanazawa 920-1192, Japan \\ ${ }^{b}$ Exploratory Materials Research Laboratories for Energy and Environment, National \\ Institute for Materials Science, Tsukuba, Ibaraki 305-0003, Japan
}

\begin{abstract}
A model active magnetic regenerator refrigerator (AMR) with the Braytonlike operation cycle was analyzed by numerical cycle simulation in the temperature range between 20 and $77 \mathrm{~K}$. In order to study the performance using magnetic material with various transition temperatures $T_{c}$, entropy of magnetic material with second order phase transition was calculated using mean field theory and Debye approximation. The cooling performance is shown to be high when the heat exhaust temperature is close to the transition temperature. It is shown that the optimized operation condition depends on both $T_{c}$ and operation temperatures. Multi-layered AMR beds were shown to improve the performance of AMR. Muti-stage AMR was also discussed.
\end{abstract}

Keywords: A: Magnetic, B: Hydrogen, E: Magnetic refrigeration

\section{Introduction}

Hydrogen energy is considered a good alternative to fossil fuels, because burning hydrogen does not produce greenhouse gases. Considering efficient, economic transport and storage, liquid hydrogen should be useful because of its high density. The key technology to use liquid hydrogen is the efficiency

\footnotetext{
*Corresponding author. Tel.: +81 76264 5669; fax: +81 762645739.

Email address: kmatsu@kenroroku.kanazawa-u.ac.jp (Koichi Matsumoto)

${ }^{1}$ Present address: Denso Corporation, 1-1 Showamachi, Kariya, Aichi 448-8661, Japan

${ }^{2}$ Present address: HIOKI E. E. CORPORATION, 81, Koizumi, Ueda, Nagano 3861192, Japan
} 
of liquefaction because the energy consumption to liquefy hydrogen is larger than $10 \%$ of the combustion energy of hydrogen. Compared to conventional liquefaction systems using Joule-Thomson expansion, magnetic refrigeration has a great potential for highly efficient hydrogen liquefaction.

Magnetic refrigeration is very attractive, since it makes use of the magnetocaloric effect and thus has the potential to be more efficient than ordinary gas expansion. The magnetocaloric effect is the thermal response of a magnetic material to a changing magnetic field. When the field is applied, magnetic entropy is reduced due to the alignment of the spins by the field. If the field is reduced adiabatically, the total entropy of the material is conserved, and the increase in magnetic entropy causes the lattice entropy to decrease. This results in a temperature decrease of the magnetic material, and is the basis of magnetic refrigeration. Magnetic refrigeration is used to achieve ultra low temperature by adiabatic demagnetization of nuclear spins. In recent years, magnetic refrigeration research has been expanded to various temperature ranges from low temperature to room temperature [1-8].

Preliminary studies showed that a magnetic refrigeration hydrogen liquefier was possible to provide reduction of capital and operation costs over gas expansion liquefiers[9]. The liquefaction of hydrogen with liquid nitrogen precooling is thought to be a possible solution for initial practical application of magnetic refrigeration. We have been developing a magnetic refrigerator system for hydrogen liquefaction that consists of liquefaction stage and precooling stage. The liquefaction and precooling stages operate Carnot and active magnetic regenerator (AMR) cycle, respectively. We have tested a magnetic refrigerator for hydrogen liquefaction stage[4, 5], and have successfully liquefied hydrogen gas that was slightly above the boiling point using Carnot cycle.

Above liquefaction temperature, a regenerative refrigeration cycle should be necessary to precool hydrogen gas, because adiabatic temperature change of magnetic material is reduced to about $2 \mathrm{~K}$ per $\mathrm{T}$ of magnetic field because of a large lattice specific heat of magnetic materials. The AMR is a regenrator-like refrigeration cycle that achieves a larger temperature span than the adiabatic temperature change of the magnetic material. The magnetically active regenerator is stuffed with magnetic material, and the regenerator matrix operates the refrigeration cycle by periodically changing magnetic fields and gas flows. We have also tested an AMR device as the precooling stage. It was confirmed that AMR cycle worked around $20 \mathrm{~K}[5]$. An AMR refrigerator was tested around $50 \mathrm{~K}$ by Astronautics group[10]. 
Cycle simulation must be used to estimate both the lowest temperature and the refrigeration capacity achieved by an AMR, because analytical calculation of the temperature variations of regenerator matrix and transfer gas is too complicated, and entropy of regenerator matrix is very dependent on both temperature and magnetic field. In the present study, a model AMR with the Brayton-like operation cycle was analyzed by numerical cycle simulation in the temperature range between 20 and 77K. AMR cycle analysis with magnetic material of $\mathrm{DyAl}_{2}$ was shown that wide temperature operation is possible with single material regenerator in the previous work[11]. However, in order to achieve operation temperature range between 20 and $77 \mathrm{~K}$, multi-layered regenerator bed or multi-stage AMR is suggested to be necessary because a magnetic material has large magnetocaloric effect only around the transition temperature. In order to study the performance using magnetic material with various transition temperatures, entropy of magnetic material with second order phase transition was calculated with mean field theory and Debye approximation. It is shown that the optimized operation condition depends on both magnetic material and operation temperatures. Multi-layered AMR beds and mutli-stage AMR were also discussed.

\section{Active magnetic regenerator (AMR) refrigerator analysis}

Active regeneration may be operable a wide temperature span because individual elements of the regenerator matrix cycle with relatively small temperature spans. Magnetic material particles are stuffed in the regenerator bed. The magnet system furnishes the regenerator matrix time-varying magnetic fields by which magnetization and demagnetization of the matrix are accomplished. And the regenerator bed is exposed to a time-varying flow of heat transfer fluid. Each segment of the regenerator bed has a unique refrigeration cycle and have interaction with the neighboring segments through the heat transfer fluid. Thus, an active regeneration system is equivalent to many cascading magnetic refrigerators. For proper cycle operation, it is essential that the transfer fluid flow is synchronized with magnetization and demagnetization of the regenerator matrix.

\subsection{Model of $A M R$}

The model AMR cycle analyzed here is the Brayton-like cycle which is the simplest cycle of an AMR and the operation was explained elsewhere[11]. The 
Brayton-like operation is divided into the following four processes; (1) Adiabatic demagnetization of regenerator bed (Field decrease causes an isentropic temperature decrease of regenerator matrix); (2) Cold flow in low magnetic field (Transfer fluid is blown from the hot end to the cold end and the thermal load is cooled); (3) Adiabatic magnetization (Field increase causes an isentropic temperature increase of regenerator matrix); and (4) Hot flow in high field (Transfer fluid is blown from the cold to the hot end and carried heat is exhausted to the hot heat reservoir). Magnetic material changes in temperature due to the magnetocaloric effect in the processes (1) and (3).

The energy conservation law yields the following governing equations with respect to gas and regenerator matrix, respectively:

$$
\begin{aligned}
A_{c} \epsilon \rho_{f} C_{f} \frac{\partial T_{f}}{\partial t}+\dot{m} C_{f} \frac{\partial T_{f}}{\partial x}= & A_{c} \epsilon k_{f} \frac{\partial^{2} T_{f}}{\partial x^{2}} \\
& +A_{c} h a_{s}\left(T_{r}-T_{f}\right)+\left|\frac{f \dot{m}^{3}}{2 d_{h} \rho_{f}^{2} A_{c}^{2}}\right|, \\
A_{c}(1-\epsilon) \rho_{r} C_{r} \frac{\partial T_{r}}{\partial t}= & A_{c}(1-\epsilon) k_{r} \frac{\partial^{2} T_{r}}{\partial x^{2}} \\
& +A_{c} h a_{s}\left(T_{f}-T_{r}\right)-A_{c}(1-\epsilon) \rho_{r} T_{r} \frac{\partial S}{\partial B} \frac{\partial B}{\partial t} .
\end{aligned}
$$

The governing equations are simplified by the following assumptions. Heat transfer fluid properties such as specific heat, density, and viscosity are constant through the regenerator and evaluated at the average temperature of the hot and cold heat reservoir. The energy change of the fluid in the void volume of the regenerator is negligible. With this assumption, first term of left side in Eq. 1 is removed. Thermal conductivity of the matrix and transfer fluid was assumed to be infinite in the direction perpendicular to flow and zero in the direction of flow. The time necessary for magnetization and demagnetization was assumed to be zero. The energy dissipation due to the viscosity of fluid is neglected. So, the fluid pressure was constant throughout the regenerator bed. The eddy current heating is negligible. After the above simplification, the governing equations in the fluid flow processes (process (2) and (4)) are rewritten as,

$$
\dot{m} C_{f} \frac{\partial T_{f}}{\partial x}=A_{c} h a_{s}\left(T_{r}-T_{f}\right)
$$




$$
A_{c}(1-\epsilon) \rho_{r} C_{r} \frac{\partial T_{r}}{\partial t}=A_{c} h a_{s}\left(T_{f}-T_{r}\right)
$$

The temperature change in the field changing processes (process (1) and (3)) are obtained only from the entropy temperature diagram of regenerator matrix.

The heat transfer coefficient is determined as follows. When the fluid flow in the regenerator is considered as that in fine tube packed with particles, the Nusselt number $(N u)$ of the matrix is related to Reynolds number $(R e)$ and Prandtl number $(P t)$ of the fluid. Heat transfer coefficient is calculated from $N u$ using the relation obtained by Quitard et al.[12].

The boundary conditions for Eqs. 3 and 4 are that the fluid enter the matrix at the temperature of the associated heat reservoir and the ends of the regenerator are insulated from the thermal conduction.

The parameter used in the simulations that is summarized in Table 1 are approximations of AMR experimental conditions of our previous study[5]. Starting with a linear temperature profile in the regenerator from the hot end to the cold end, steady state was achieved when the relative temperature change of each regenerator segment and out coming transfer fluid were less than $1 \times 10^{-4} \mathrm{~K}$.

Table 1: A summary of the AMR parameter

\begin{tabular}{l|rl}
\hline Maximum field & 5.0 & Tesla \\
Length of AMR bed & 0.1 & $\mathrm{~m}$ \\
Diameter of the AMR bed & 0.045 & $\mathrm{~m}$ \\
Diameter of magnetic material & 0.4 & $\mathrm{~mm}$ \\
Packing factor of AMR bed & 0.64 & \\
Period for each transfer fluid flow & 2.0 & $\mathrm{sec}$ \\
Heat transfer fluid & He gas $0.5 \mathrm{MPa}$ & \\
\hline
\end{tabular}

\subsection{Model of magnetic material}

A model magnetic material is necessary to study the influence of the material on the performance of AMR. Rare earth intermetallic compounds are good refrigerants because of their large magnetic moments. $R T_{2}$ ( $R$ : rare earth; $T: \mathrm{Al}, \mathrm{Ni}$ ) are Laves-phase compounds with a cubic $\mathrm{MgCu}_{2}$ structure. These compounds exhibit second order phase transition from para- to ferromagnetic states and have been studied as magnetic refrigerant[13]. The 
transition temperature can be controlled in the temperature range between 20 and $80 \mathrm{~K}$ by choosing the rare earth element $R$ or metal $T$. Then, magnetic materials with various transition temperatures of second order phase transition from para- to ferromagnetic state was used as a model material in this study. The Lande $g$ factor and total angular moment $J$ of rare earth atom is assumed as that of dysprosium. Hereafter, magnetic material that has $T_{c}$ at $x \mathrm{~K}$ is written as Tc $x$.

Entropy of magnetic material consists of magnetic and lattice component in the temperature range of this study. In order to calculate entropy temperature diagram, the magnetization of the model material $M(T)$ was calculated as functions of temperature using mean field theory. The entropy change of material $\Delta S$ was evaluated from temperature dependent magnetization $M(T)$ using the following Maxwell relation,

$$
\Delta S(T, H)=\int_{0}^{H}\left(\frac{\partial M}{\partial T}\right)_{H} d H .
$$

The magnetic entropy changes $\Delta S$ of the model materials induced by the field change from 0 to $5 \mathrm{~T}$ obtained from the above calculation are presented in Fig. 1.

The lattice component of entropy was calculated using Debye approximation. Debye temperature of model material was assumed to be $310 \mathrm{~K}$ that is typical for $R T_{2}$ compounds[14]. The entropy temperature diagram of model material of Tc60 is in very good agreement with that of $\mathrm{DyAl}_{2}$. So, our model magnetic material is appropriate to use in the cycle analysis.

\section{Results and Discussion}

\subsection{Single layer $A M R$ bed}

Figure 2 shows the heat load per cycle as a function of mass flow rate of transfer fluid, $\dot{m}$ for the model material Tc60. The operation temperature range was between 77 and $65 \mathrm{~K}$. Heat load $Q_{L}$ increases with $\dot{m}$ and has a maximum. Then, $Q_{L}$ decreases with $\dot{m}$. When $\dot{m}$ is small, $Q_{L}$ becomes small because transfer fluid can carry small heat. $Q_{L}$ decreased with increasing $\dot{m}$ in large $\dot{m}$ region. The temperature of the transfer fluid increased at the cold end with large $\dot{m}$ because the temperature distribution in the regenerator bed was disturbed by too large heat capacity of transfer fluid. As a result, AMR has an optimum $\dot{m}$. In Fig. 2, $Q_{L}$ for $\mathrm{DyAl}_{2}$ is also shown and agrees quantitatively with that for Tc60. This represents the adequacy of our model. 


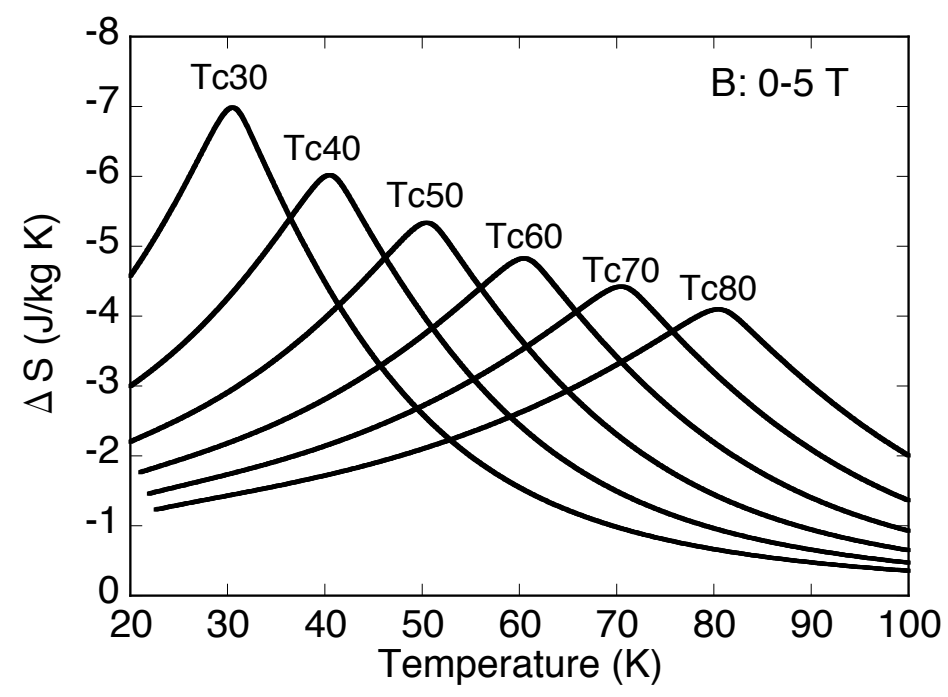

Figure 1: Magnetic entropy change of model materials with various transition temperature $T_{c}$ induced by $5 \mathrm{~T}$ field, as calculated using mean field theory. Each curve respectively corresponds to $\Delta S$ of Tc30, Tc40, Tc50, Tc60, Tc70, and Tc80 from low temperature.

Figure 3 shows heat load as a function of $\dot{m}$ for materials Tc70 and Tc50. The operation temperature span is $25 \mathrm{~K}$ for both cases but temperature range is between 80 and $55 \mathrm{~K}$ for Tc70 and between 55 and $30 \mathrm{~K}$ for Tc50. As shown in Fig. 3, the optimum $\dot{m}$ is different between materials and temperature range. This is ascribed to the lattice specific heat. Large $\dot{m}$ gives rise to a large disturbance in the temperature profile of regenerator bed during cold and hot flow, and decreases in $Q_{L}$. This effect have larger influence in low temperatures because lattice specific heat becomes smaller with decreasing temperature as is explained by Debye theory. Then, the optimum $\dot{m}$ for low temperature operation becomes small. In order to decrease the disturbance in temperature profile, magnetic materials which have large specific heat is desirable. Magnetic materials which have low Debye temperature and/or Schottky specific heat due to appropriate excitation energy level will have ability to improve the refrigeration performance.

AMRs using single magnetic material with various $T_{c}$ were simulated in the case that hot heat reservoir temperature $T_{H}$ was set at $77 \mathrm{~K}$. The optimum operation conditions were obtained for various materials and cold heat reservoir temperatures for AMRs with Tc60, Tc70, and Tc80 by changing 


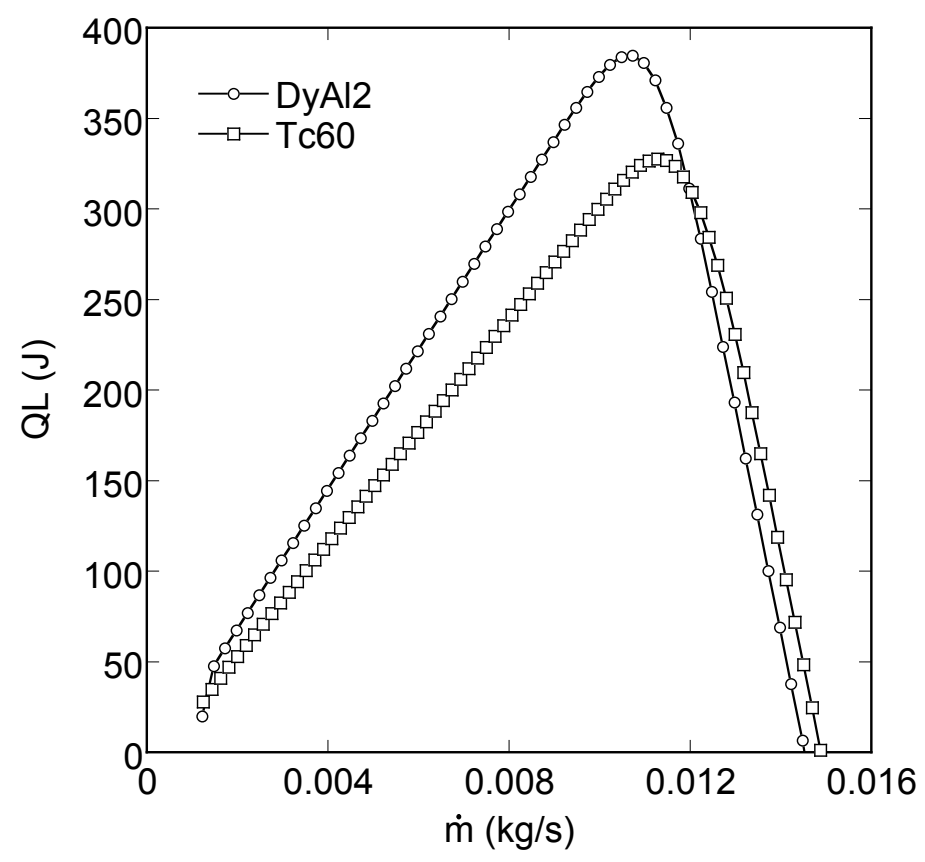

Figure 2: Heat load of AMR using material with Tc60 as a function of $\dot{m}$. That using $\mathrm{DyAl}_{2}$ is also represented for comparison. The operation temperature range is between 77 and $65 \mathrm{~K}$.

$\dot{m}$. $Q_{L}$ and coefficient of performance, COP in the optimum condition are plotted as functions of operation temperature span in Fig. 4. $Q_{L}$ and COP decrease as monotonic functions of temperature span. AMR bed with Tc70 has the largest $Q_{L}$. As for COP, AMR bed with Tc80 has the largest value. As shown in Fig. 1, Tc70 has the largest total amount of $\Delta S$ in the operation temperature range between 60 and $77 \mathrm{~K}$ and Tc60 has the largest $\Delta S$ in the temperature range between $55 \sim 77 \mathrm{~K}$ and $50 \sim 77 \mathrm{~K}$. The large $\Delta S$ is naively thought to give large $Q_{L}$. However, the AMR bed with Tc60 has the smallest $Q_{L}$ and COP. This represents that large $\Delta S$ does not simply mean high refrigeration performance. When magnetic material exchanges heat by magnetic field change at a constant temperature, the exchanged heat is expressed as temperature times entropy change $T \Delta S$. So, Carnot efficiency will be obtained if the entropy changes are the same at cold and hot heat reservoir temperature in an ideal thermal cycle. In real refrigeration cycles, exhausted heat at hot reservoir is larger than that of an ideal cycle due to ir- 


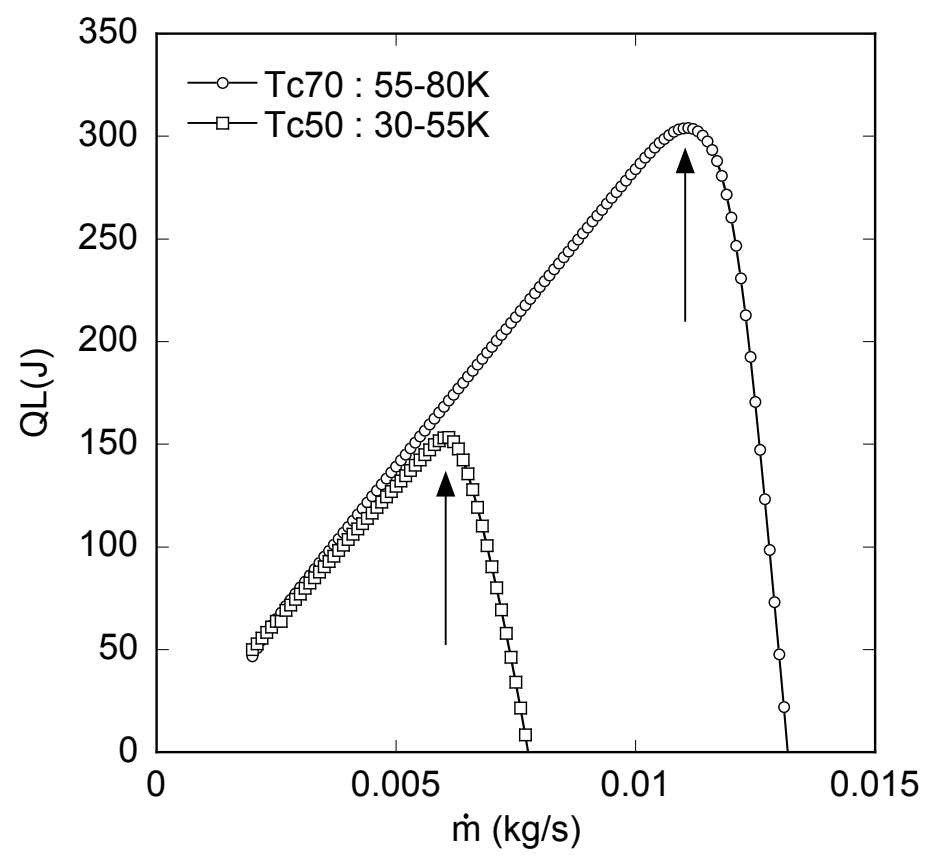

Figure 3: Heat load of AMR using single material of Tc70 and Tc50 as functions of $\dot{m}$. The operation temperature span is $25 \mathrm{~K}$ for both cases but temperature range is between 80 and $55 \mathrm{~K}$ for Tc70 and between 55 and $30 \mathrm{~K}$ for Tc50. Arrows represent the optimum $\dot{m}$.

reversibility of the cycle. Temperature variation of $\Delta S$ is important to carry heat from cold to hot side. When $\Delta S$ at high temperature side is small, amount of magnetic material which works to exhaust heat should increase and only small fraction of AMR bed could absorb heat from cold reservoir. Then, $Q_{L}$ obtained with this material cannot be as large as that expected by $\Delta S$ at low temperature. It is shown in Fig. 4 that temperature dependence of $\Delta S$ also has significant effect on COP. As shown in Fig. 1, $\Delta S$ of Tc80 increases with increasing temperature below $80 \mathrm{~K}$ and this material is thought to transfer entropy efficiently to hot heat reservoir.

\subsection{Two layered $A M R$ bed}

It is shown that $\Delta S$ has significant effect on the performance of AMR. $\Delta S$ of Tc80 at $50 \mathrm{~K}$ is about half of that at peak temperature $(80 \mathrm{~K})$ and only about half of that of Tc60 at $50 \mathrm{~K}$. In order to achieve large temperature 

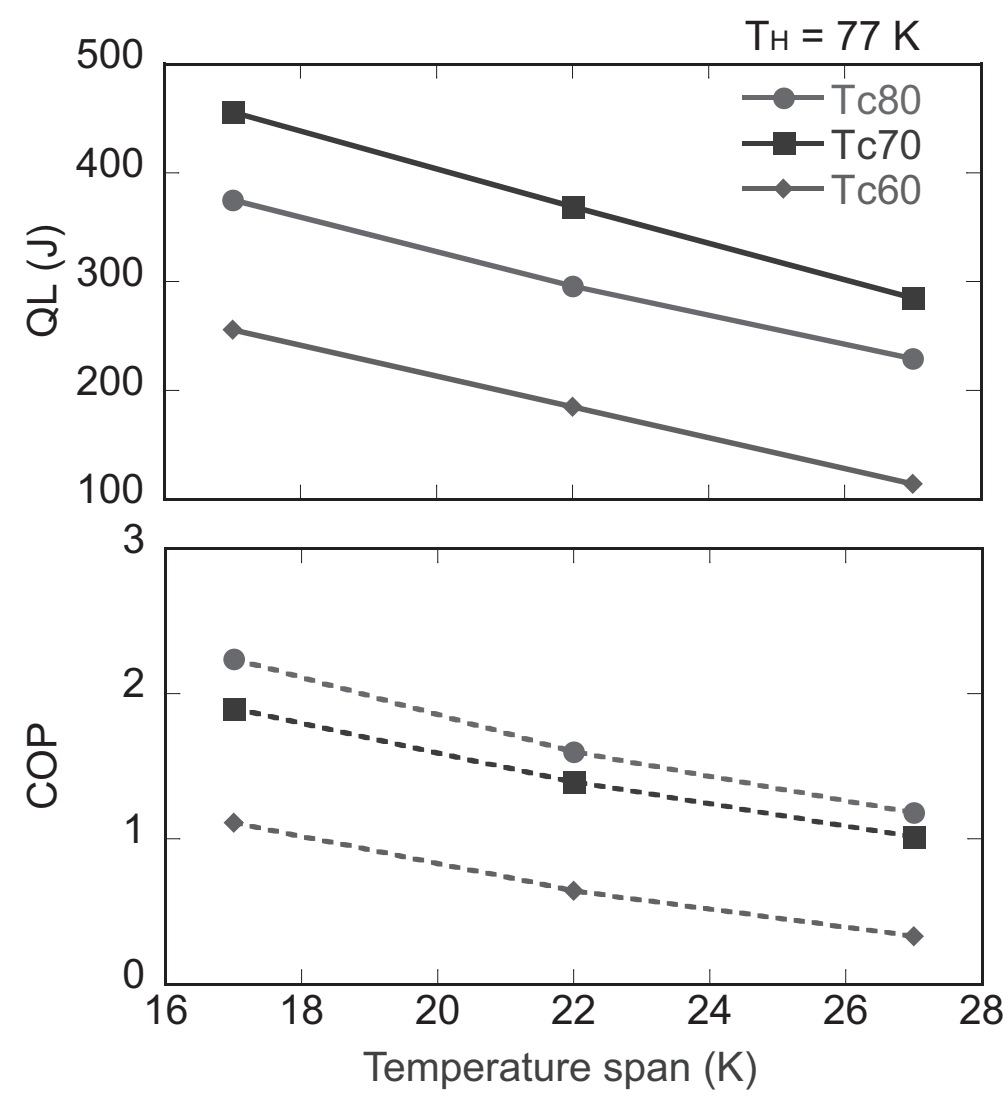

Figure 4: Heat load and COP of AMR with single magnetic material as functions of operation temperature span. Performance with Tc80, Tc70, Tc60 are compared. $T_{H}$ is 77 K.

span, multi layered AMR bed with low $T_{c}$ at low temperature side is expected to have high performance. We have analyzed two layered AMR with various $T_{c}$ and ratio between materials. Results for the two layered AMR bed with Tc80 and Tc60 is represented in Fig. 5. $Q_{L}$ is increased when Tc80 is replaced by Tc60 into the low temperature side. For the purpose of improving $Q_{L}$, Fig. 5 shows that there is an optimum substitution ratio of about $30 \%$ in all the operation temperature. However, COP decreases with increasing Tc60 ratio.

We have simulated in various conditions. It was found that two layered AMR bed was able to improve the performance in some conditions and the 

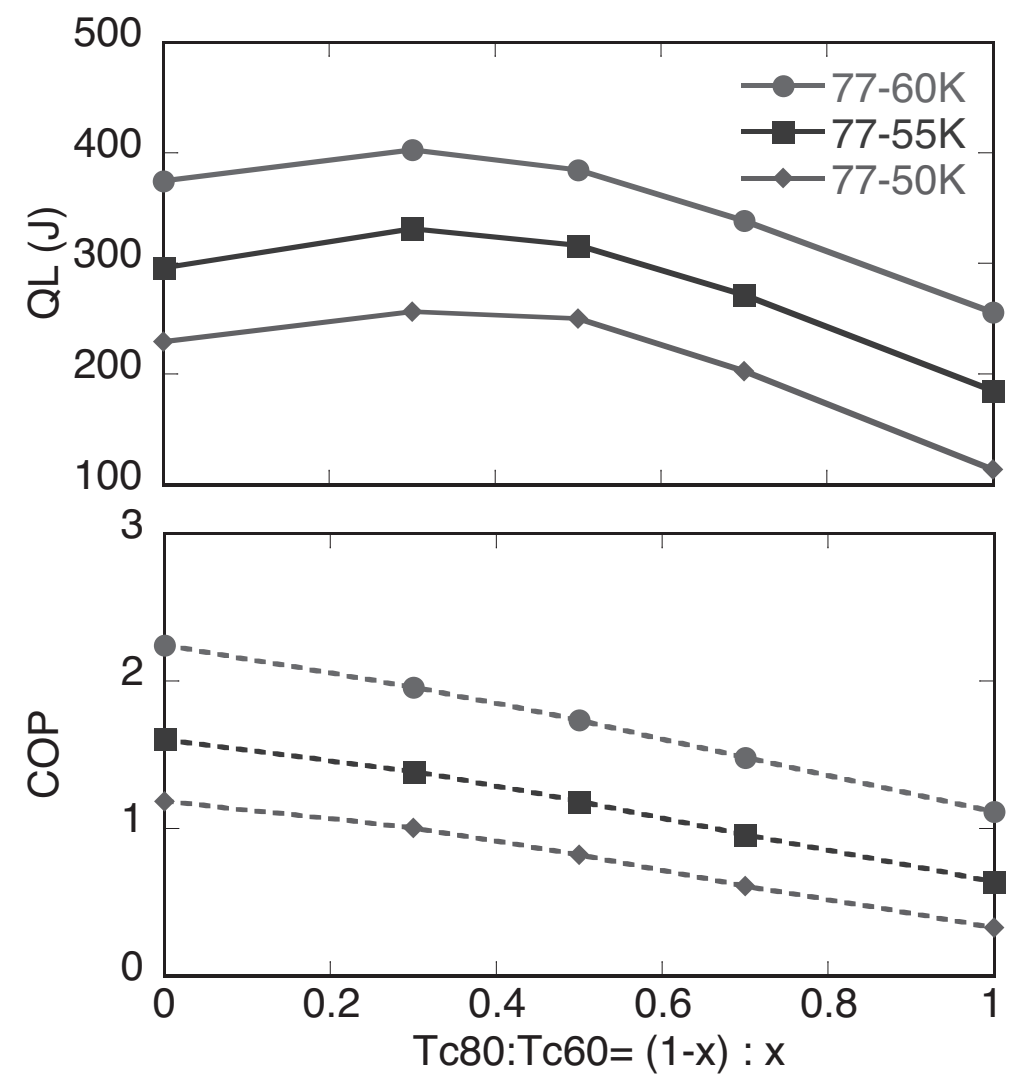

Figure 5: Heat load and COP of two layered AMR with Tc80 and Tc60 as functions of ratio between the two materials. Results for $T_{L}=60,55$, and $50 \mathrm{~K}$ are shown. $T_{H}$ is 77 $\mathrm{K}$.

optimum condition depends on many parameters such as operation temperature range, $T_{c}$ of materials and so on, even in the simple AMR model of this study. This means that detailed cycle analysis is necessary to have proper operation for a practical refrigerator. In general, increase of low $T_{c}$ material at low temperature side doesn't always give rise to an improvement. Probably similar situation of single layer AMR bed occurs. Magnetic material in the high temperature side looks to play an important role in order to transfer entropy produced by irreversibility from low temperature side to high heat reservoir. 


\subsection{Multi-stage AMR}

Multi-stage AMR with various $T_{c}$ is another way to expand the operation temperature span and improve performance. This is thought to be useful especially in low temperatures as discussed below. As shown in Fig. 3, the optimum $\dot{m}$ is different between magnetic material and temperature range. Each material in multi layered AMR bed is thought not to operate in optimum condition because the $\dot{m}$ is constant through one AMR bed. From this point of view, optimum operation of whole system is possible in the case of multistage AMR. In our preliminary analysis, two stage AMR can have several times larger $Q_{L}$ than single AMR when appropriate materials and connection temperature are selected.

In order to optimize the operation of AMR system, there are so many parameters such as number of AMR stage, layers in one AMR bed, transition temperatures of materials, and so on. In this study, size and aspect ratio of AMR bed, particle size of magnetic material, and strength of magnetic field were not discussed, that should have large effects. We focused mainly on the influence of $T_{c}$ of magnetic material. Thus, further detailed analysis is indispensable to understand AMR. For example, temperature dependent properties of transfer fluid, viscosity should be considered in the next step. Magnetic materials with first order phase transition is known to have large $\Delta S$. Cycle simulation using materials with first order transition will be important. Other cycles such as Ericsson cycle will be necessary to analyze to reveal more about AMR system.

\section{Conclusions}

A simplified AMR model was analyzed by numerical cycle simulation using model magnetic materials. This model has been shown useful to understand multilayered AMR and multistage AMR system. Double layered AMR bed which has different $T_{c}$ was shown that could improve the performance of AMR. The optimum $\dot{m}$ is shown to vary when the operation temperature range is different. It was pointed out that cycle analysis plays an important role in understanding AMR operation since many parameters have significant effects. 


\section{Acknowledgments}

Financial support provided by NEDO in the project of Search and Research of Innovative and Leading Technologies, is greatly appreciated. We appreciate to Dr. K. Kamiya for his contribution in early stage of numerical calculation.

[1] Giauque WF, MacDougall DP. Attainment of Temperatures Below $1^{\circ}$ Absolute by Demagnetization of $\mathrm{Gd}_{2}\left(\mathrm{SO}_{4}\right)_{3} \cdot 8 \mathrm{H}_{2} \mathrm{O}$. Phys Rev $1933 ; 43(9): 768$.

[2] Hakuraku Y, Ogata H. A rotary magnetic refrigerator for superfluid helium production. J Appl Phys 1986; 60(9):3266-8.

[3] Numazawa T, Hashimoto T, Nakagome H, Tanji N, Horigami O. The helium magnetic refrigerator. II - Liquefaction process and efficiency. Adv Cryog Eng 1984;29:589-596.

[4] Numazawa T, Kamiya K, Yoshioka S, Nakagome H, Matsumoto K. Development of a magnetic refrigerator for hydrogen liquefaction. AIP Conf Proc 2008;985(1):1183-1189.

[5] Matsumoto K, Kondo T, Yoshioka S, Kamiya K, Numazawa T. Magnetic refrigerator for hydrogen liquefaction. J Phys Conf Ser 2009;150(1):012028.

[6] Jimm CB, Jastrab A, Sternberg A, Pecharsky VK, Gschneider Jr KA, Osborne M, Anderson I. Description and performance of a near-room temperature magnetic refrigerator, Adv Cryog Eng 1998;43:1759-1766.

[7] Matsumoto K, Ito T, Hashimoto T. An Ericsson magnetic refrigerator for low temperature. Adv Cryog Eng 1988;33:743-50.

[8] Hirano N, Nagaya S, Takahashi M, Kuriyama T, Ito K, Nomura S. Development of magnetic refrigerator for room temperature application. Adv Cryog Eng 2002;47:1027-1034.

[9] Utaki T, Nakagawa T, Yamamoto T, Kamiya K, Numazawa T. Research on a Magnetic Refrigeration Cycle for Hydrogen Liquefaction. Cryocooler 2007;14:645. 
[10] Zimm CB, Johnson JW, Murphy RW. Test results on a 50K magnetic refrigerator. Adv Cryog Eng 1996;41(B):1675-1681.

[11] Matsumoto K, Hashimoto T. Thermodynamic analysis of magnetically active regenerator from 30 to $70 \mathrm{~K}$ with a Brayton-like cycle. Cryogenics 1990;30(10):840-845.

[12] Quintard M, Kaviany M, Whitaker S. Two-medium treatment of heat transfer in porous media: Numerical results for effective properties. Adv in Water Resources 1997;20(2-3):77-94.

[13] Hashimoto T, Matsumoto K, Kurihara T, Numazawa T, Tomokiyo A, Yayama H, Goto T, Sahashi M. Investigations on the possibility of the $\mathrm{RAl}_{2}$ system as a refrigenrant in an Ericsson type magnetic refrigerator. Adv Cryog Eng 1986;32:279-286.

[14] von Ranke PJ, Pecharsky VK, Gshneidner Jr KA, Pecharsky VK, Lograsso TA, Schlagel DL. Magnetic properties of single-crystal Dy $\mathrm{Al}_{2}$. Phys Rev B 2005;72(2):024403. 


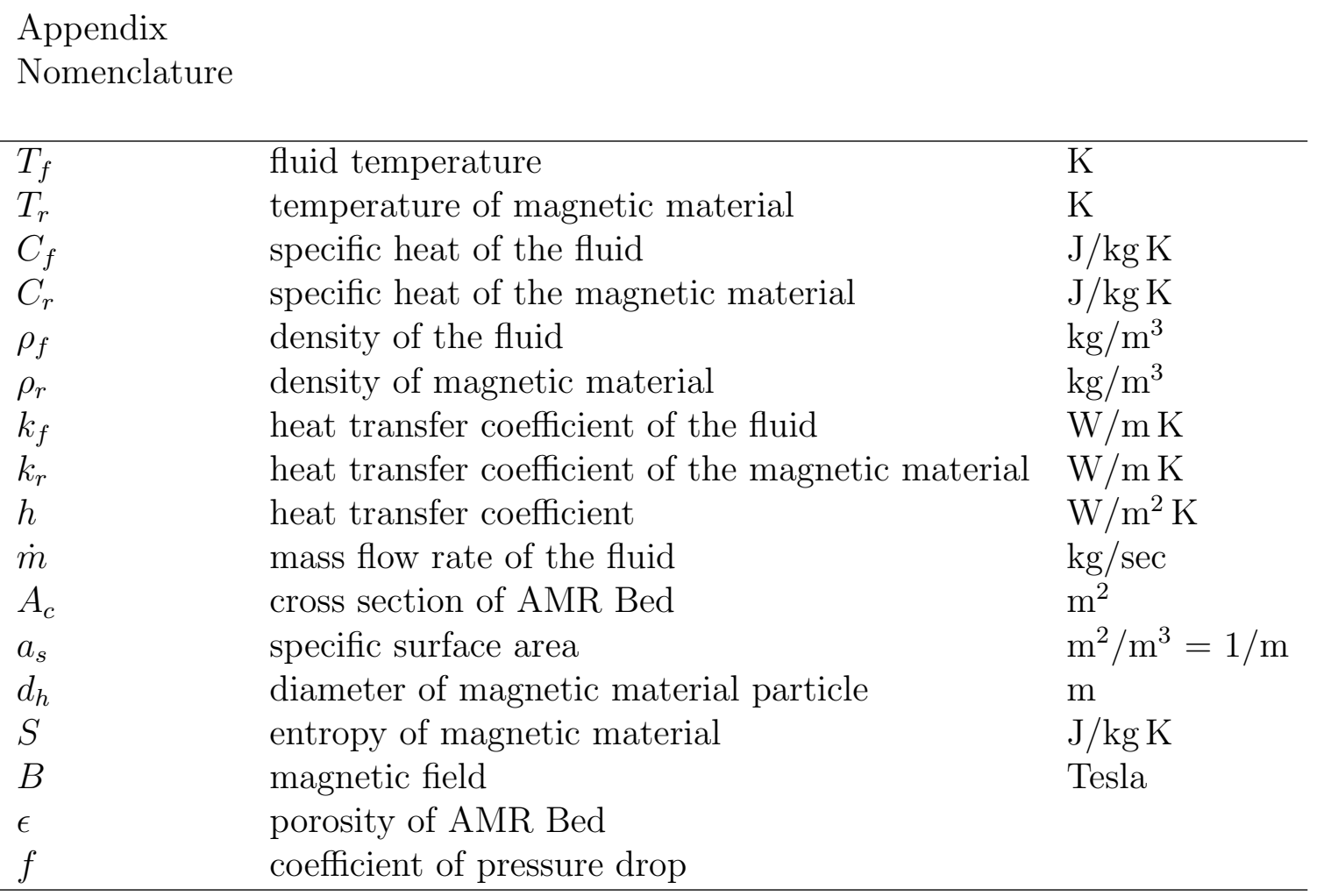

